\title{
Positioning Inventory in Clinical Trial Supply Chains
}

October 20, 2012

\begin{abstract}
As a result of slow patient recruitment and high patient costs in the United States, clinical trials are increasingly going global. While recruitment efforts benefit from a larger global footprint, the supply chain has to work harder at getting the right drug supply, to the right place, at the right time. Certain clinical trial supply chains, especially those supplying biologics, have a combination of unique attributes that have yet to be addressed by existing supply chain models. These attributes include a fixed patient horizon, an inflexible supply process, a unique set of service-level requirements, and an inability to transfer drug supplies among testing sites. In this paper, we provide a new class of multi-echelon inventory models to address these unique aspects. The resulting mathematical program is a nonlinear integer programming problem with chance constraints. Despite this complexity, we develop a solution method that transforms the original formulation into a linear integer equivalent. By analyzing special cases and numerically studying a hypothetical real-life example, we develop novel insights into inventory positioning in clinical trial supply chains. We also study the impact of site network on the supply chain cost and the trade-off between inventory overage and the expected recruitment time.
\end{abstract}

Keywords and Phrases: Clinical trial supply chain, multi-echelon inventory model, finite patient horizon.

\section{Introduction}

Clinical trials, as mandated by the United States Food and Drug Administration (FDA), seek to test the safety and efficacy of an investigative drug in human subjects. Given the drug's absence of an established safety record, potential patients are understandably leery of enrolling in these trials. In the United States, these patients tend to favor already approved treatment options. Not surprisingly, patient recruitment is a typical bottleneck in conducting clinical trials and one study suggests that $80 \%$ of clinical trials are failing to meet their patient recruitment deadlines (Getz and 
de Bruin 2000). To counter the slow patient recruitment and high costs of enrolling patients in the United States, clinical trials are increasingly going global in their search for patients (Rowland 2004). In quantifying this globalization, Thiers, Sinskey and Berndt (2008) report the following data on the growth in the number of clinical trial sites (the doctors' offices and hospitals that are used to enroll patients in a clinical trial) for various countries:

\begin{tabular}{lc}
\hline Country & Annual Growth Rate (\%) \\
\hline China & 47.0 \\
Russia & 33.0 \\
Argentina & 26.9 \\
Czech Republic & 24.6 \\
Mexico & 22.1 \\
\hline United States & -6.5 \\
\hline
\end{tabular}

Table 1: Growth in Number of Offshore Clinical Trial Sites.

We see from Table 1, that clinical trial growth in emerging markets far outpaces that in the United States. In fact, the United States is experiencing declines in the number of trial sites being used. In addition to the relocation of trials, more clinical trials are seeking participants in multiple countries simultaneously. In $2005,7.8 \%$ of the trials reported in three top medical journals were being conducted in 10 or more countries while in 1995, none of the articles in those journals reported such high levels of globalization (Glickman, et al. 2009).

In addition to facing the complexity of an increasingly global supply chain, clinical trial supply managers are held accountable for very high service levels. Supply managers fear being the cause of delaying a trial because of drug shortages. Delaying a trial's completion by even one day means a one day loss of patent exclusivity and the associated revenue (Roberts, Lynch and Chabner 2003). As a result, “[clinical supply managers] knowingly make twice as many drugs as they're going to ever need because they want to make sure there is no patient any place in the world that is ready to be dosed that doesn't have a drug" (Powell 2010). Thus, significant inventory is kept at globally dispersed clinical trial sites to guarantee these near $100 \%$ service levels. Once a trial concludes, usually determined by achieving some predetermined patient enrollment target, all leftover inventory is essentially wasted. Despite this, grossly over-producing drug supply is often considered a viable option and large inventory overages (i.e. production in excess of the expected enrollment) are commonplace.

To see how serious the inventory overage issue may be in practice, let's consider a clinical 
trial which plans to enroll 612 patients (this example is stylized from the 612-patient, 45-site trial described in Le Chevalier, et al. 1994). If there is only one site, shipping 612 drug kits to the site ensures a 100\% fill rate. To accelerate patient recruiting, 45 sites can be opened, but imposing a typical $99 \%$ fill rate for the first 612 patients requires shipping 1035 kits to the sites (assuming inventory is carried only at sites and patient recruitment follows a Poisson process with independent but identical arrival rates at all sites). The planned overage is $423 \mathrm{kits}$, about $70 \%$ of the required kits.

Recently, huge overages are being scrutinized. Clinical supply managers are being asked to cut costs as clinical supply costs can potentially account for a significant portion (e.g., 40\%, see Fleischhacker 2009 for examples) of the clinical trial spending. With large pharmaceutical companies like Bristol Meyers Squibb spending roughly $\$ 200$ million per year on clinical supplies, they estimate that reducing planned overages is a way to save $\$ 40$ million annually (Powell 2010). As eroding pharmaceutical profits are a fact of life for the industry, reducing overages is a logical response to budgetary pressures; the goal of the clinical supply manager is to do more with less. Patrick Vallance, Senior Vice President - Medicines Discovery and Development at GlaxoSmithKline (GSK), describes GSK's migration away from using large inventory overages in clinical trials (Vallance 2011):

Four years ago, it was the norm when ordering clinical trial supplies to have an overage of over $100 \%$ sometimes $200 \%$. Statistical and mathematical modeling show that you can reduce that overage to under $50 \% \ldots$ [Using those modeling tools, our overages went] from an average of well over 100\% [in 2006] to something under 50\% [in 2009] resulting in $\$ 120$ million of drug substance savings. This late-stage focus on these efficiencies is incredibly important when running these large clinical studies.

As suggested in the above quote, mathematical models are becoming instrumental tools to support the inventory needs of global trials on tighter budgets. Yet despite this, very little modeling (other than simulation) that accommodates the unique elements of clinical trial supply chains is available in academic or practitioner literature. The question of how to manage inventory to efficiently support a global clinical trial is largely unanswered. In this paper, we study a class of clinical trial supply chains and address their unique requirement by a new class of multi-echelon inventory models. 


\subsection{Clinical Trial Supply Chain - An Example}

For large global clinical studies, clinical trial supply chains are highly complex and no two clinical trials are run in exactly the same manner. We present disguised details of a trial that a large U.S. based pharmaceutical company conducted and for which relevant data has been provided. The trial tested the effectiveness of an antibiotic in treating a specific type of infection and may be deemed typical for Phase III testing of biologic drugs. The trial's patient horizon (i.e. the target number of patients to be recruited) was 600 and the patient recruitment was accomplished in nine months. During the trial, each patient received one clinical trial package (i.e. drug supply, packaging, and labeling) and all treatments were administered intravenously in a hospital or doctor's office.

Previously collected drug stability data supported a 24-month shelf life for the investigational drug and thus, drug expiration was not a concern for this trial.

The supply chain for this trial started with active pharmaceutical ingredient (API) production, formulation and packaging, all completed in Europe. Distribution from the central warehouse in Italy to 32 trial sites world-wide was accomplished via multiple country depots. Using country depots is typical and Alex Klim (2010) of DHL Supply Chain's Clinical Trial Logistics Service succinctly states the reasoning as applied when shipping to Brazil, Russia, India and China (BRIC):

... the vast majority of large and medium sized pharmaceutical manufacturers and biotechs still like to manufacture their research and development (R\&D) products in their European and North American production plants. This invariably means applying for an import license and having to go through the BRIC country's importation processes to supply a local depot. Although it is not completely impossible to operate without it, it is highly advisable to have a local depot to store bulk medical supplies in each of the BRIC countries; this is largely due to the long lead times around the importation process, or in some cases due to local regulations.

Transit times from central warehouse to country depots were on the order of a few weeks, consisting mainly of regulatory clearance time as opposed to actual transportation time. Transit times from depots to sites were on the order of a few days.

Traditionally, inventory of investigative drugs is often held at trial sites to account for the requirements of recruited patients without much resupply from the depots and central warehouse (Peterson, Byrom, Dowlman, McEntegart 2004). An alternative is to pull back some inventory to the depots and central warehouse, and resupply the sites as needed. The question is, how should 
we position inventory in the clinical trial supply chain to ensure the desired drug availability in the most cost efficient manner? To answer this question, we must account for the unique features of clinical trial supply chains.

\subsection{Clinical Trial Supply Chains - Unique Features}

Clinical trial supply chains resemble spare part supply chains; they have similar network structure and each seeks to satisfy demand that is random, infrequent ${ }^{1}$, and only met at the lowest echelon of the supply chain. However, clinical trial supply chains require new mathematical models due to many unique attributes:

- A Finite Patient Horizon, $S$ : Each clinical trial has a predefined recruitment target, $S$, which is the necessary sample size for the study. $S$ is part of a protocol that is specified prior to trial commencement and deviations from the protocol will jeopardize the acceptance of trial results by the FDA. Once the target is reached, recruitment is closed and no more patients will be enrolled in the trial. ${ }^{2}$

- Inflexible Supply: Due to statistical consistency considerations and/or the significant fixed costs associated with the production and quality control of investigational drugs, companies often produce once for the entire trial prior to its commencement. This is particularly true for biologics that are produced using biology-based processes (as opposed to chemical processes) such as vaccines and many cancer therapies. For biologic trials, only a very small number of production lots can be used so that statistical consistency, in regards to efficacy and safety of the different lots, can be maintained. For example, Pederson et al (2007) study the effectiveness of a human papillomarvirus vaccine by randomizing patients to receive drug supply from one of four specific production lots. All lots were manufactured prior to administering the vaccine in any trial participants. In addition, with the regulatory and testing requirements imposed by the FDA, it is considered desirable, when possible, to have a drug of uniform quality that was produced all in one batch or in one run (see Oncolytics Biotech 2003 for an example).

- Inability of Cross-Shipping: By regulation, site-to-site shipping is discouraged and must "remain the exception" due to possible safety and trial quality issues (European Commission

\footnotetext{
${ }^{1}$ As described in $\S 6$, the anticipated enrollment rate at any one site is often much less than one patient per day.

${ }^{2}$ Since the safety and efficacy of the drug are being tested, it makes sense to cease new exposures to the drug until results are evaluated.
} 
2003). In Andrews' (2004) analysis of the strategic value of clinical supplies, he notes that "in practice, the delays and potential for mistakes in retrieving a batch of material from one site and re-releasing and/or transferring to another can, and does, make this course of action impractical." In fact, study sponsors often impose "standard operating procedures [which] prohibit the transfer of study drug from one site to another." (Barnett International 2010). Cross shipping from one country depot to another country depot is also not recommended because of customs issues, the need for relabeling, and potential risks of disqualifying a trial due to poor regulatory adherence regarding drug accountability.

- Two Stringent Service-Level Requirements: A clinical trial supply chain manager is tasked with achieving high immediate fill rates at sites and an even higher patient fill rate for the entire trial. The immediate fill rate measures the percentage of patients at a site who are administered the investigative drug immediately upon arrival. The patient fill rate measures the percentage of patients entering the trial who are eventually administered the drug. In other words, the patient fill rate measures the percentage of patients rejected from the trial because the corresponding sites run out of stock and the system exhausts all inventory available to resupply the sites.

Because a rejection of a patient or a delay of service due to clinical drug shortages is considered unacceptable, the system must have sufficient inventory for the first $S$ (the sample size) patients regardless of which site they arrive to, and it is also desirable that drug supply is immediately available to those patients. In practice, the trial is designed to achieve a patient fill rate of $100 \%$ or nearly $100 \%$, and very high immediate fill rates (often exceeding 99\%). The $100 \%$ patient fill rate ensures that each site can continue recruiting patients until the patient enrollment target is reached and thus, minimizes the recruitment time. Practitioners often adopt these service level requirements as a proxy for their time-to-market performance because estimating the cost of time is difficult due to uncertainties in the trial outcome, the drug's market potential, and the opportunity cost of time and money not being available to support investment in another drug's clinical trial.

These unique attributes, combined with a complex logistics network, inherent demand uncertainty (due to random enrollments at sites and randomization of patients), and relatively high shipping costs, make a global clinical-trial supply chain difficult to manage. In absence of more sophisticated modeling techniques, companies produce multiples of baseline forecasts as planned overage (McDonnell and Mooraj 2009). However, this overage is essentially a costly waste that 
ultimately leads to paying for the destruction of expensive materials. Clearly, the initial inventory position in the system can have a significant impact on the overage and service levels due to the inability of cross-shipping among sites and country depots. Thus, one of the main challenges in the clinical trial supply chain is to achieve the required service levels with the minimum supply chain cost by making intelligent inventory decisions.

\subsection{Summary of Results}

In this paper, we investigate the inventory decisions made within clinical trial supply chains. Specifically, we determine the optimal upfront production quantity, the optimal inventory positions, and the optimal shipping quantities to minimize the system-wide inventory overage and shipping costs while achieving the desired immediate fill rates at the sites and the desired patient fill rate for the trial $(\S 1.2)$.

We first present a new class of multi-echelon inventory models incorporating the unique aspects of the clinical trial supply chain. We then develop a solution method that transforms the nonlinear integer programming problem with chance constraints to a linear integer equivalent. The transformation relies on one of the unique aspects of clinical trial supply chains, the "finite patient horizon", to generate a "finite" set of deterministic constraints equivalent to the stochastic chance constraints. The transformation can be done in a pseudo-polynomial time in the patient horizon and shipping quantities. By analyzing a few special cases and numerically studying a hypothetical real-world example, we show that the optimal stock positions in clinical trial supply chains differ qualitatively from the classical multi-echelon inventory literature and the tradition in practice, and thus can lead to significant savings. Finally, we study the impact of site network on supply chain costs and also, the trade-off between the planned inventory overage and the expected recruitment time.

\section{Literature Review}

A good introduction to the challenges of managing a global clinical trial supply chain can be found in Lis, Gourley, Wilson, and Page (2009) where it is succinctly noted that "the key challenge clinical trial supply chain (CTSC) managers face in global distribution is ensuring that supplies arrive at the trial sites on time and in good condition." To be on time, the inventory not only must be produced in sufficient quantity to meet demand, but must also be properly positioned in the supply 
chain to satisfy demand as it is realized.

The management of inventory in these supply chains has attracted limited attention from industry, and has attracted even less attention from academia. In industry literature, it is usually advocated that inventory control policies in the clinical trial supply chain are selected using simulation (see Abdelkafi, Beck, David, Druck and Horoho 2009 and Peterson, Byrom, Dowlman and McEntegart 2004 and references therein). In contrast, this is the first paper that we know of to present an analytical model for inventory control in clinical trial supply chains. While analytic models generally have the drawbacks of simplifying assumptions, the assumptions we make here are typical and consistent with our motivating example. Some notable similarities and differences between our model and simulation studies include:

- Dosage Level Changes: In some trials, various patients may be assigned to different dosing levels of the investigational drug and the dosage levels being tested may change over the course of the trial. This is an example of an adaptive trial and would be atypical, but supported by simulation. Most trials are still traditional with dosage being set prior to the trial starting. Our motivating example and analytical model are consistent with the traditional trial approach.

- Continuous Review Inventory Policy: It is often assumed within these simulation models that an integrated voice response system (IVRS) is available so that inventory can be monitored continuously in time (McEntegar and O'Gorman 2005) and as inventory at a location falls below a specified trigger, more inventory is ordered. Our paper also adopts this assumption.

- Inventory Policy as Model Output: The output of our model is an inventory policy for a given set of service levels. In contrast, simulation studies require the inventory policy as input and then simulate the service level performance. Thus, prior to deciding an inventory policy, simulation models require multiple combinations of inventory policies to be tested with each one requiring multiple (in the thousands) Monte Carlo simulations to ensure compliance with the near $100 \%$ service levels as required by clinical trials.

- Perishability: Unlike our motivating example where shelf-life is not an issue, many clinical trials must deal with the issue of perishability. We recognize that simulation is better equipped at handling shelf-life concerns and that analytic modeling of inventory systems with perishable products often require "analysis of multidimensional stochastic processes, and fairly involved computations, due to the rapid growth of dimensionality of the process under investigation." 
(Ravichandran 1995)

Despite the availability of sophisticated tools for simulation in the real-world, inventory policies (e.g., the trigger levels, shipping quantities) in clinical trial supply chains are still often set using experience and by looking at the patterns from previous clinical trials. Going forward, we can envision that both analytic models and simulation models will be used with more frequency. In fact, it is highly desirable to combine the two by using analytic models to generate initial input for simulation models when more detailed modeling assumptions are required (software tools like BioClinica Optimizer also advocate this combined approach).

Sophisticated models have been developed to manage inventory in distribution networks (e.g., for spare parts). However, these models have yet to find their way to the problems faced by clinical trials and yet, it is noted that these models are needed. Shah (2004) points out many of the key challenges faced by pharmaceutical supply chains and surveys the literature that addresses those challenges. Of particular interest is the recent focus of academicians on capacity planning for clinical trial supply. Our work differs from this surveyed work in that we assume the allocation of capacity to support a trial has already taken place and we now focus on the more operational/tactical policy details of managing trial inventory.

Within the context of multiechelon inventory research, our models are most closely related to work done for service parts where inventory is reviewed continuously in time (see reviews by Zipkin 2000, Muckstadt 2005 and Simchi-Levi and Zhao 2011). One seminal work in this stream of literature is Sherbrooke (1968). He approximated the distribution of backorders at the depot by its first moment in a two-echelon distribution system when one-for-one ordering policies are used. Improving on this approximation is the two-moment approximation by Graves (1985) who shows how to effectively approximate backorder and lead time demand by a negative binomial distribution. Svoronos and Zipkin (1991) refine the approximation by Graves (1985) and extend it to evaluate multi-echelon distribution systems.

Graves (1985) and Axsater (1990) provide means to exactly evaluate the distribution of net inventory levels in a multi-echelon distribution system, but these methods require the convolution of multiple probability distributions and thus are computationally intensive. Simchi-Levi and Zhao (2005) extend the exact approach to evaluate tree structure supply chains subject to fill rate constraints, but in making the stock positioning decisions, approximations in line with Graves (1985) and Svoronos and Zipkin (1991) are utilized.

All aforementioned work focuses on one-for-one ordering policies. Batch ordering policies, which 
account for economies of scale, also received considerable attention in the literature. Zipkin (1986) specifies conditions under which a single-stage batch ordering inventory system can be transformed into a set of base-stock inventory systems. Axsater (1993) shows how to evaluate a multi-echelon distribution system with batch ordering polices exactly and approximately. For other recent work on evaluation and/or optimization of stock positioning in distribution systems for service parts, we refer to Caglar, Li, and Simchi-Levi (2004) and Caggiano, Jackson, Muckstadt, and Rappold (2007). In this paper, we utilize the results of Graves (1985) and Zipkin (1986) to approximate certain subsystems within a three-echelon supply chain that includes one central warehouse, multiple country depots, and multiple sites, where some sites may be supplied directly by the central warehouse and others via country depots.

The stock positioning problem in clinical trial supply chains represents a new variation of the classical multi-echelon inventory models because it is unique in three key aspects: 1) system performance concerns, such as immediate fill rates, are only relevant before the target number of patients is recruited, 2) production is done prior to the commencement of the trial, and 3) clinical trial supply chains cannot afford to reject patients due to supply shortages and therefore have to place enough stock in the system to satisfy all recruited patients up to the patient horizon (i.e., ensure $100 \%$ patient fill rate). These differences result in non-trivial modifications to the objective functions and constraints used in classical models, and lead to new models, solutions and insights.

\section{Modeling and Formulation}

In this section, we consider a clinical trial supply chain of a general distribution topology, depicted in Figure 1, where a central warehouse $(\mathrm{CW})$, indexed as 0, supplies multiple country depots, indexed by $i=1,2, \ldots, I$, and some sites directly, which are indexed by $0 j$ where $j=1,2, \ldots, J_{0}$. The other sites are satisfied via a particular depot $i$ and are indexed $i j$ where $j=1,2, \ldots, J_{i}$. For convenience, we call the subsystem of country depot $i$ and its corresponding sites $i j$ the $\operatorname{depot} i$ subsystem.

\subsection{Assumptions and Notation}

Throughout this paper, we make the following assumptions.

Assumption 1 (a) The clinical study has a finite patient horizon, S. (b) Each patient requires one package or vial which is produced prior to the commencement of the trial. (c) Inventory (i.e., 


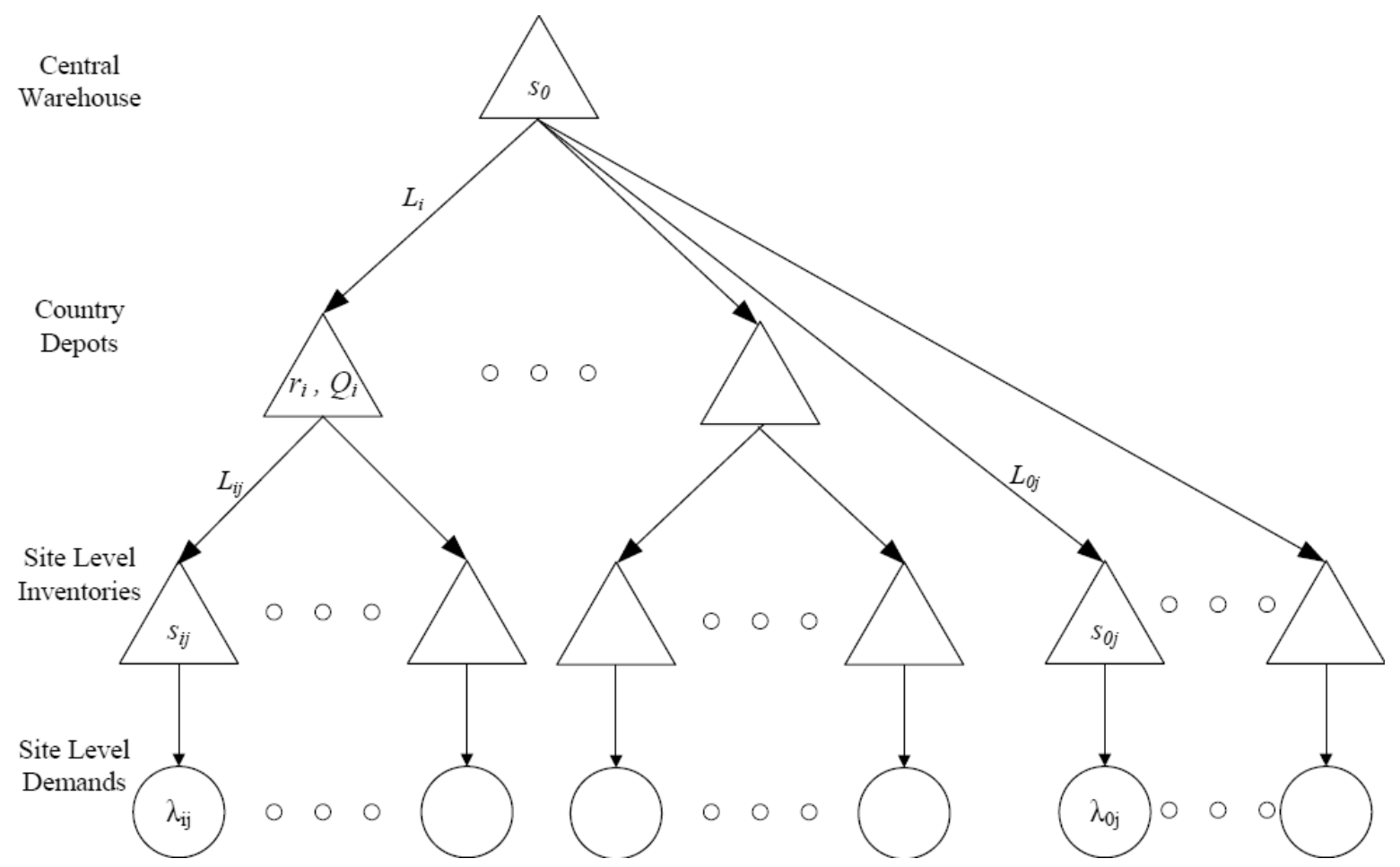

Figure 1: A general clinical trial supply chain.

dosages of drug in either package or vial form) cannot be transferred among sites or among country depots. (d) Patient recruitment occurs only at trial sites and follows an independent Poisson process. (e) Patients recruited at one site are unable to seek treatment from other sites. (f) In the event of a temporary site-level stockout (with replenishment in-transit), patients will still enroll in the trial and wait for resupply. (g) The patient fill rate is $100 \%$, i.e., the system guarantees supply for the first $S$ patients whichever site they arrive to. (h) The transit times (i.e for shipping and regulatory clearance) between $C W$ and depots, $C W$ and direct sites, and depots and sites are constant. (i) The shipping costs from depots (or the CW) to testing sites (or direct sites) are negligible.

The first four assumptions are justified in $§ 1.1-1.2$. The assumption of a Poisson recruitment process (1d) is widely used in the industry literature, see Abdelkafi et al. (2009) and references therein. Clinical sites (e.g., hospitals, doctor's offices) are chosen based on their ability to enroll patients who meet the eligibility requirements of the trial. The recruitment rates can be estimated based on the track record of patients visiting each site previously and/or data of previous trials for the same disease. Assumption (1e) is a reflection of the geographical dispersement of clinical trials. In reality, Assumption (1f) may or may not hold depending on the type of disease, the urgency of treatment, and the location of trial sites. In this paper, we consider the case where patients can and will wait for resupply if the site runs into a temporary drug shortage. Assumption (1g) 
is justified in $§ 1.2$. The assumption of constant lead times (1h) is for simplicity and practitioners have indicated that transit times may not be a significant source of variability.Assumption (1i) is based on the fact that the costs associated with shipping from depots (or CW) to sites (or direct sites) are often negligible as compared to those from CW to depots due to custom clearance and a much larger geographic distance of the latter.

Consistent to the motivating example $(\S 1.1)$, we assume that the clinical trial supply chain works as follows: production of trial drug is done before the trial starts; inventory can be carried by the testing sites, the depots and the CW; and lastly, sites are supplied by depots which are supplied by the CW until the CW runs out of stock. In addition,

Assumption 2 (a) Inventory at each site is controlled by a continuous-review base-stock policy. (b) Inventory at each country depot is controlled by a continuous-review batch ordering policy.

The assumption of a continuous-review inventory policy (2a and $2 \mathrm{~b})$ at sites and depots closely represents modernized clinical trial supply chains. Through the use of integrated voice response systems (IVRS), all doctors administering clinical trial drugs to patients are required to call in to a system for instructions on which drug package is to be administered to the patient. Through this system, both real-time inventory information is maintained and automated shipments to replenish site-level inventory can be triggered (Byrom 2002). While sometimes it will take multiple demands at a site to trigger an order, we make a simplifying assumption (2a), that is appropriate in high value biologic trials, where an order is triggered each time a demand occurs.

We define the following notation:

\section{System Parameters:}

- $\lambda_{i j}, \lambda_{0 j}$ : Patient recruitment rates at sites $i j$ and $0 j$ respectively; $\lambda_{i}=\sum_{j=1}^{J_{i}} \lambda_{i j}$.

- $L_{i}, L_{i j}, L_{0 j}$ : Lead times from CW to depot $i$, from depot $i$ to site $i j$, and from CW to direct site $0 j$, respectively.

- $D_{i}(L), D_{i j}(L), D_{0 j}(L)$ : Demand during lead time $L$ at depot $i$, sites $i j$, and sites $0 j$ respectively.

\section{Decision Variables:}

- $s_{i j}, s_{0 j}$ : Base-stock levels at sites $i j$ and $0 j$ respectively.

- $r_{i}, Q_{i}$ : The reorder point and order quantity at depot $i$. 
- $s_{0}$ : The initial stock at the CW, note that the CW cannot be replenished.

- $x_{i}$ : The expected number of shipments to depot $i$ for the entire trial (excluding the initial shipment).

- $y_{i}$ : The expected number of drug packages shipped to depot $i$ for the entire trial (excluding the initial shipment).

\section{Costs and Service Levels:}

- $c$ : The cost of a drug package.

- $K_{i}$ : The fixed cost of a shipment from the CW to depot $i$.

- $v_{i}$ : The variable shipping cost from the CW to depot $i$.

- $\zeta$ : The target patient fill rate for the trial.

- $\zeta^{\prime}$ : The target immediate fill rate at each site.

Our objective is to minimize system-wide inventory overage and shipping costs subject to a $100 \%$ patient fill rate $(\zeta=100 \%$, by Assumption $1 \mathrm{~g})$ and a high immediate fill rate $\left(\zeta^{\prime}\right)$, by setting inventory levels $\left(s_{0}, r_{i}, s_{i j}, s_{0 j}\right)$ and shipping quantities $\left(Q_{i}\right)$ appropriately.

\subsection{The Role of The Finite Patient Horizon}

The finite patient horizon, $S$, plays an important role in the patient fill rate requirement, $\zeta=$ $100 \%$, which guarantees a sufficient supply for the first $S$ patients arrived in the trial. Under this requirement, $s_{0}$ can be determined by $S$ and other decision variables $r_{i}, Q_{i}, s_{i j}$ and $s_{0 j}$ for all $j$. We make the following assumptions.

Assumption 3 (i) Each site starts with an initial inventory level equal to its base-stock level, depot $i$ starts with an initial inventory level $r_{i}+Q_{i}$, for all $i$. (ii) If the $C W$ has insufficient stock to meet the order quantity of a depot, then the depot takes whatever the CW has to offer.

Part (i) of Assumption 3 models real-world practice that it is more economical to fully stock the depots and sites initially to achieve the scale economies in shipping. Part (ii) of Assumption 3 can be justified by observing that if otherwise, the CW may have to carry more inventory than required just to meet a depot's order quantity. 
To ensure $\zeta=100 \%$ while minimizing the inventory at the central warehouse, we must set

$$
s_{0}=S-\min \left\{\min _{i}\left\{r_{i}+Q_{i}+\min _{j} s_{i j}\right\}, \min _{j} s_{0 j}\right\}
$$

To see this, we first observe that at the time when the CW runs out of stock, the system can still satisfy $\min \left\{\min _{i}\left\{r_{i}+q_{i}+\min _{j} s_{i j}\right\}, \min _{j} s_{0 j}\right\}$ many future patients without rejecting anyone due to insufficient stock of the system. This can be easily seen by considering the worst-case scenario of demand only arriving through one site. This observation implies that to guarantee $\zeta=100 \%$ for the entire trial, we should have at least $S-\min \left\{\min _{i}\left\{r_{i}+q_{i}+\min _{j} s_{i j}\right\}, \min _{j} s_{0 j}\right\}$ many patients already recruited by the time the $\mathrm{CW}$ runs out of stock. We also note that each order of size $Q_{i}$ placed by depot $i$ implies $Q_{i}$ patients already being recruited by this depot subsystem (by Assumption 3 ). Thus, the total orders filled by the CW at any time must not exceed the total number of patients recruited thus far. Excluding the $Q_{i}-q_{i}$ patients from $S$ because they are satisfied by depot $i$ at the time CW stocks out, we arrive at Eq. (1). Lastly, $s_{0} \geq 0$ because setting $\min _{j} s_{0 j}>S$ or $r_{i}+Q_{i}+\min _{j} s_{i j}>S$ for all $i$ is clearly suboptimal.

By the finite patient horizon, it is also logical to assume the following stopping rules: (i) Site $0 j$ (or $i j$ ) stops ordering when the total number of patients left to be recruited is no more than its base-stock level $s_{0 j}$ (or $s_{i j}$ ). (ii) Depot $i$ stops ordering when the number of patients left to be recruited is no more than $r_{i}+\min _{j} s_{i j}$. By Eq. (1), the $\mathrm{CW}$ will never stockout before a direct site or a depot stops ordering because when the CW runs out stock, Eq. (1) ensures that each direct site/depot subsystem alone has enough stock to cover all the patients left to be recruited.

\subsection{Shipping Costs and Model Formulation}

We now calculate $x_{i}, y_{i}$ and the expected shipping cost to depot $i$. By the stopping rule of $\S 3.2$, we only need to consider the first $S-\left(r_{i}+\min _{j} s_{i j}\right)$ patients recruited. The proof of the following lemma is given in the Appendix.

Lemma 4 Suppose that among the first $S-\left(r_{i}+\min _{j} s_{i j}\right)$ patients recruited, $N_{i}$ patients are recruited by the depot $i$ subsystem, then the number of orders placed by depot $i$ after the trial starts, $X_{i}$, satisfies the following inequalities:

$$
\left\lfloor\frac{N_{i}}{Q_{i}}\right\rfloor \geq X_{i} \geq\left\lceil\frac{N_{i}}{Q_{i}}\right\rceil-1
$$

and the absolute difference between these bounds and $X_{i}$ is at most 1. 
The expected number of shipments to depot $i, x_{i}=E\left[X_{i}\right]$. An exact characterization of $x_{i}$ involves highly nonlinear terms such as the $k$-combination of the $S-\left(r_{i}+\min _{j} s_{i j}\right)$ patients, $\left(\underset{k}{S-\left(r_{i}+\min _{j} s_{i j}\right)}\right)$, which renders an efficient optimization hardly possible. Thus, we seek a simple approximation as a surrogate for $x_{i}$ with a guaranteed performance. First, we use the lower bound, $\left\lceil\frac{N_{i}}{Q_{i}}\right\rceil-1$, for $X_{i}$, then we switch the sequence of expectation and ceiling to arrive at

$$
\left\lceil\frac{E\left[N_{i}\right]}{Q_{i}}\right\rceil-1=\left\lceil\frac{\left[S-\left(r_{i}+\min s_{i j}\right)\right] \frac{\sum_{j} \lambda_{i j}}{\sum_{i, j} \lambda_{i j}+\sum_{j} \lambda_{0 j}}}{Q_{i}}\right\rceil-1 .
$$

The difference between Eq. (3) and $x_{i}$ is at most 2 because the absolute difference between $\left\lceil\frac{E\left[N_{i}\right]}{Q_{i}}\right\rceil$ and $E\left[\left\lceil\frac{N_{i}}{Q_{i}}\right\rceil\right]$ is at most 1 .

Given Eqs. (1) and (3), the problem of minimizing inventory overage and shipping cost subject to the fill-rate constraints $\left(\zeta\right.$ and $\left.\zeta^{\prime}\right)$ can be formulated as follows,

$$
\begin{array}{ll}
\min & c\left[s_{0}+\sum_{i}\left(r_{i}+Q_{i}+\sum_{j} s_{i j}\right)+\sum_{j} s_{0 j}-S\right] \\
& +\sum_{i}\left[K_{i}+v_{i}\left(r_{i}+Q_{i}+\sum_{j} s_{i j}\right)\right]+\sum_{i}\left[K_{i} x_{i}+v_{i} y_{i}\right] \\
\text { s.t. } & \text { immediate fill rate at site } i j \geq \zeta^{\prime}, \quad \forall i j, \\
& \text { immediate fill rate at site } 0 j \geq \zeta^{\prime}, \quad \forall 0 j, \\
& Q_{i} \leq \bar{Q}, \quad \forall i, \\
& y_{i}=x_{i} Q_{i}, \quad \forall i, \quad \forall i, \\
& {\left[S-\left(r_{i}+\min _{j} s_{i j}\right)\right] \frac{\sum_{j} \lambda_{i j}}{\sum_{i j} \lambda_{i j}+\sum_{j} \lambda_{0 j}}-Q_{i} \leq x_{i} Q_{i}, \quad} \\
& s_{0}=S-\min _{i}\left\{\min _{i}\left\{r_{i}+Q_{i}+\min _{j} s_{i j}, \min _{j} s_{0 j}\right\}, \quad \forall i, j .\right.
\end{array}
$$

The first term of the objective function, Eq. (4), is the inventory overage cost, where the inventory overage is the difference between the total inventory at the beginning of the trial (i.e., inventory at the CW, plus inventory at depots, plus inventory at sites) less the patient horizon. The second term of the objective function is the cost of shipping the initial inventory to the depot subsystems. The third term is the expected shipping cost to the depots after the trial starts. Eq. (7) comes from the size of the container $(\bar{Q})$ being used for shipping. ${ }^{3}$ Eq. (9) is based on Eq. (3), and Eq. (10) comes from Eq. (1).

\footnotetext{
${ }^{3}$ Often the quantities being shipped to the country depots are limited by the size of the container being used for air freight from the central warehouse. For example, Envirotainer (http://www.envirotainer.com) manufactures containers to provide a temperature controlled environment required for many clinical trial materials.
} 
Problem $(P 0)$ differs from the classical multi-echelon inventory models that minimize inventory and shipping costs subject to fill-rate constraints by the constraint on $s_{0}$ (Eq. 10) and the shipping cost calculations. These special features come from the unique attributes of the clinical trial supply chain - the finite patient horizon, the inflexible supply process, the system-wide patient fill rate requirement, and the inability to cross-ship. The constraint on $s_{0}$ effectively connects the inventory decisions at the CW and all depot subsystems.

\subsection{Steady-State Approximation}

We now characterize the immediate fill rate constraints (Eqs. 5-6) in Problem ( $P 0$ ). Due to the finite patient horizon, the operational characteristics of the clinical trial supply chain, e.g., immediate fill rates at sites, are transient. However, such a system in transient state can be properly approximated by its steady-state counterpart if the patient horizon is significant and the patient recruitment period is much longer than the replenishment lead times. Fortunately, this is often true in practice because the recruitment period for a Phase II or Phase III clinical trial is typically measured in months and years while the lead times are typically measured in days or weeks (Kohner 2010). For instance, in our motivating example (see §1.1), the recruiting period is nine months while the replenishment lead times are 1-2 weeks at most.

For the depot-supplied sites, by Eq. (1) and the fact that the CW will never stockout before a depot stops ordering, we can approximate the immediate fill rate at site $i j$ (Eq. 5) in Problem (P0) by its steady-state counter-part (see, e.g., Zipkin 1986, Axsater 1993, Simchi-Levi and Zhao 2011). Thus Eq. (5) can be written as follows:

$$
\operatorname{Pr}\left\{s_{i j}-B_{i j}\left(r_{i}, Q_{i}\right)>D_{i j}\left(L_{i j}\right)\right\} \geq \zeta^{\prime}, \quad \forall i j,
$$

where $B_{i j}$ is the number of backorders that depot $i$ owes site $j$ in steady state, depending on $r_{i}$ and $Q_{i}$. Specifically, $B_{i j}$ follows a binomial distribution with $N=B_{i}$ and $p=\lambda_{i j} / \lambda_{i}$ where $B_{i}=\left[D_{i}\left(L_{i}\right)-I P_{i}\right]^{+}$and $I P_{i}$, the inventory position at depot $i$, follows a uniform distribution in $\left\{r_{i}+1, r_{i}+2, \ldots, r_{i}+Q_{i}\right\}$.

The steady-state approximation, Eq. (11), is in one sense conservative and in another less so. It is conservative because actual steady-state fill rates at testing sites are slightly higher than $\zeta^{\prime}$. The employed approximation is based on a randomized initial condition (see for example, Song 2000) and in our model, the trial starts with full inventory at all depots (i.e., the initial inventory at depot $i$ is $r_{i}+Q_{i}$ by Assumption 3 (i)). Thus, with a higher initial inventory position, depots provide better drug availability to the sites than what the steady-state performance suggests. The 
approximation is less conservative because Assumption 3 (ii) may also impact the actual fill rate during the transient period near the end of the trial, but this would only apply to the last few patients arriving at most to one depot subsystem (i.e. the subsystem encountering this assumption's exception). Hence, the negative impact of using a steady-state approximation on actual fill rates is limited.

For sites supplied directly, we can use a similar logic to approximate the immediate fill rate constraint at direct site $0 j$ (Eq. 6) by its steady-state counter-part (see, e.g., Simchi-Levi and Zhao 2011):

$$
\operatorname{Pr}\left\{s_{0 j}>D_{0 j}\left(L_{0 j}\right)\right\} \geq \zeta^{\prime}, \quad \forall 0 j
$$

Problem $(P 0)$ is a nonlinear integer program with chance constraints as specified by Eqs. (11)(12). The trade-off is intuitively clear: the high immediate fill rates and high fixed shipping costs mandate inventory being spread out to testing sites. On the other hand, the high drug cost and high variable shipping costs favor centralizing inventory at the $\mathrm{CW}$ and/or depots. Problem $(P 0)$ provides a mathematical framework to achieve the optimal balance.

\subsection{Preliminary Results}

The following lemma explores the dependence among the stock decisions at different locations.

Lemma 5 In the optimal solution to Problem (P0), $s_{i j}$ is the minimum value to ensure the immediate fill rate constraint at site ij given $\left(r_{i}, Q_{i}\right)$ and unlimited supply from the $C W$; $s_{0 j}$ is the minimum value to ensure the immediate fill rate constraint at site $0 j$ given unlimited supply from the $C W$.

Proof. We first note that the CW never stocks out before depot $i$ stops ordering by Assumption (1g). Thus, we can treat the $\mathrm{CW}$ as if it has unlimited supply. Suppose the claim on $s_{i j}$ is not true, then there must exist an optimal solution to Problem $(P 0)$ such that the new solution of reducing $s_{i j}$ by one and increasing $r_{i}$ by one while keeping everything else unchanged still remains feasible for all fill rate constraints. In this new solution, the $x_{i}, y_{i}$ and $s_{0}$ can only decrease while everything else remains the same. Thus, the objective function can only decrease. We now have a better solution which creates a contradiction to the optimality claim of the original solution. The proof is completed for $s_{i j}$.

For $s_{0 j}$, we note that by the constraint of $s_{0}$, the $\mathrm{CW}$ will never stockout before a direct site stops ordering. By the same logic as that of $s_{i j}$, we can prove the desired result for $s_{0 j}$. 


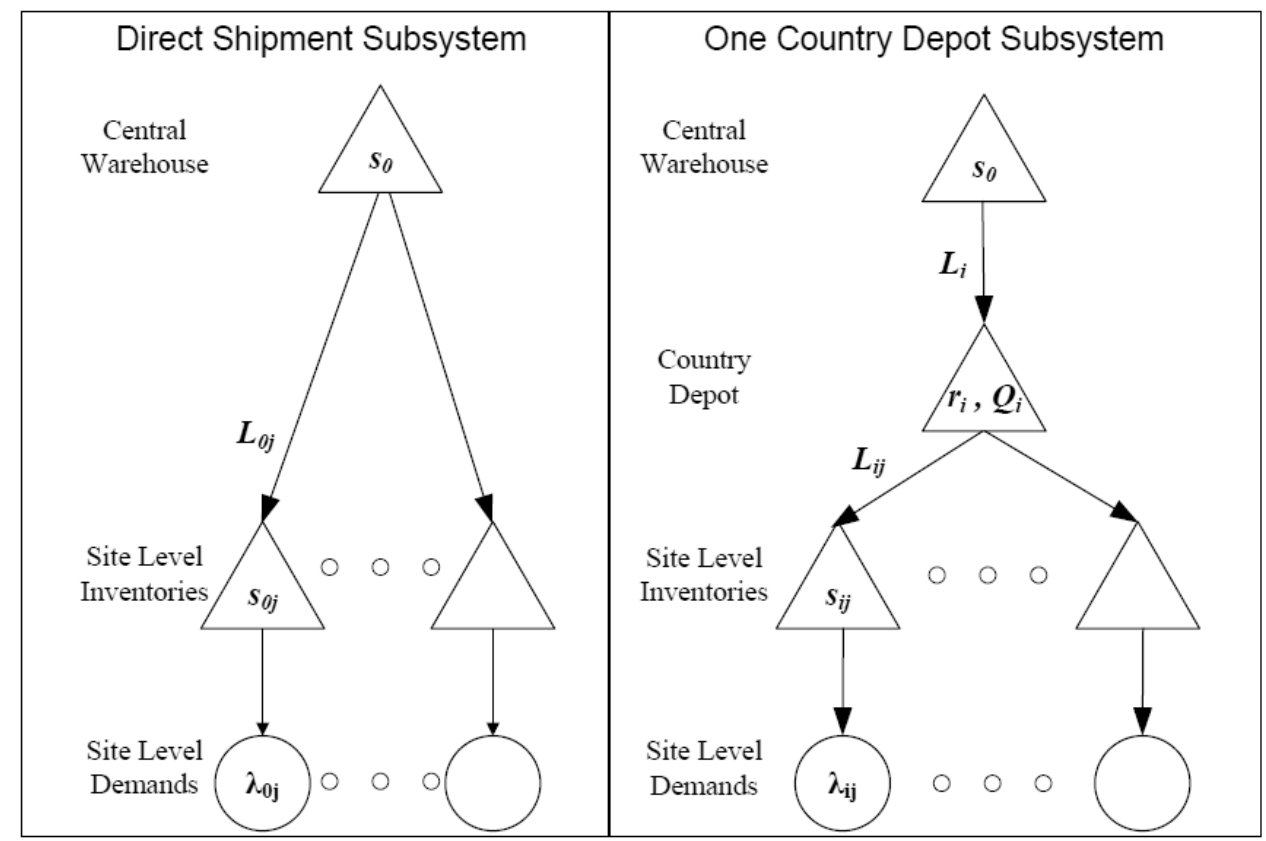

Figure 2: The direct shipment network vs. the network with one depot subsystem.

Lemma 5 implies that for a direct site, $s_{0 j}$ can be solved independently of all depot subsystems; for a depot subsystem, the decision variables $\left(r_{i}, Q_{i}\right)$ and $s_{i j}$ are dependent.

\section{Analysis of Special Cases}

In this section, we study two special cases of the clinical trial supply chain: (i) all sites are supplied directly by the central warehouse, (ii) all sites are supplied by one country depot which in turn is supplied by the central warehouse (see Figure 2). These cases represent two basic elements of the general network, and their analysis can help us develop analytical insights on inventory positioning in the general network. To this end, we ignore shipping costs and set all shipping quantities to 1.

For case (i), the direct shipment network, Problem $(P 0)$ reduces to:

$$
\begin{array}{lll}
\min & \left\{c\left[s_{0}+\sum_{j} s_{0 j}-S\right]\right\} \\
\text { s.t. } & \operatorname{Pr}\left\{s_{0 j}>D_{0 j}\left(L_{0 j}\right)\right\} \geq \zeta^{\prime}, \quad \forall 0 j \\
& s_{0}=S-\min _{j} s_{0 j} \\
& s_{0}, s_{0 j} \text { are nonnegative integers, } \quad \forall j .
\end{array}
$$


Replacing $s_{0}$ in the objective function by its constraint yields,

$$
\begin{array}{ll}
\min & \left\{c\left[\sum_{j} s_{0 j}-\min _{j} s_{0 j}\right]\right\} \\
\text { s.t. } & \operatorname{Pr}\left\{s_{0 j}>D_{0 j}\left(L_{0 j}\right)\right\} \geq \zeta^{\prime}, \quad \forall 0 j \quad \\
& s_{0}, s_{0 j} \text { are nonnegative integers, } \quad \forall j .
\end{array}
$$

By Lemma 5, an optimal solution is to choose the smallest $s_{0 j}$ that achieves the desired fill rate at site $0 j$ for all $0 j$ given unlimited supply from the CW. This means that we should minimize inventory at sites subject to the fill rate constraints.

For case (ii), the one country depot $(i)$ system, Problem $(P 0)$ reduces to:

$$
\begin{array}{lll}
\min & \left\{c\left[s_{0}+r_{i}+1+\sum_{j} s_{i j}-S\right]\right\} & \\
\text { s.t. } & \operatorname{Pr}\left\{s_{i j}-B_{i j}\left(r_{i}, 1\right)>D_{i j}\left(L_{i j}\right)\right\} \geq \zeta^{\prime}, & \\
& s_{0}=S-r_{i}-1-\min _{j} s_{i j} \\
& s_{0}, r_{i}, s_{i j} \text { are nonnegative integers, } & \forall j .
\end{array}
$$

To simplify further, we place the constraint, $s_{0}=S-r_{i}-1-\min _{j} s_{i j}$, into the objective function, then we have an equivalent problem:

$$
\begin{array}{ll}
\min & \left\{c\left[\sum_{j} s_{i j}-\min _{j} s_{i j}\right]\right\} \\
\text { s.t. } & \operatorname{Pr}\left\{s_{i j}-B_{i j}\left(r_{i}, 1\right)>D_{i j}\left(L_{i j}\right)\right\} \geq \zeta^{\prime}, \quad \forall j, \\
& r_{i}+1+\min _{j} s_{i j} \leq S \\
& r_{i}, s_{i j} \text { are nonnegative integers, } \quad \forall j .
\end{array}
$$

Proposition 6 identifies the optimal solution to Problem (16), see the Appendix for a proof.

Proposition 6 The optimal solution to the following problem,

$$
\begin{array}{ll}
\min & \left\{c\left[\sum_{j} s_{i j}\right]\right\} \\
\text { s.t. } \quad & \operatorname{Pr}\left\{s_{i j}-B_{i j}\left(r_{i}, 1\right)>D_{i j}\left(L_{i j}\right)\right\} \geq \zeta^{\prime}, \quad \forall j, \\
& r_{i}+1+\min _{j} s_{i j} \leq S \\
& r_{i}, s_{i j} \text { are nonnegative integers, } \quad \forall j, \quad
\end{array}
$$

is also optimal for Problem (16).

Proposition 6 reveals the optimal inventory positioning: To minimize the system-wide inventory, one must minimize site level inventory. This can be done by stocking as much inventory at the depot $i$ as possible to reduce the needed stock at sites. Effectively, one can increase $r_{i}$ (to achieve a lower $s_{i j}$ ) as long as $s_{0}$ stays non-negative. 
Both special cases indicate that we should pull inventory from sites back to the depot and/or $\mathrm{CW}$ as much as possible subject to the immediate fill rate constraints at the sites. Such a strategy differs from the multi-echelon inventory literature (see, e.g., Graves 1996) and the tradition in clinical trials (Peterson, et al. 2004) which suggest to hold most of the safety-stock at the sites.

The special cases allow us to gain insights into general networks with multiple depot subsystems. Consider a general network and suppose that in the optimal solution, depot $i$ subsystem achieves $\min \left\{\min _{i}\left\{r_{i}+1+\min _{j} s_{i j}\right\}, \min _{j} s_{0 j}\right\}$ in Eq. (10). Then, the optimal stocking strategy is to minimize the total inventory at all depot subsystems except for depot $i$ where one must minimize the site level inventory. However, we do not know in advance which depot achieves the minimum in Eq. (10) before we solve the Problem $(P 0)$. Thus, it is not trivial to make the optimal inventory decisions for a general clinical trial supply chain, and it is even harder if we must also consider shipping costs and shipping quantities.

\section{Solution Methods}

Problem $(P 0)$ is an integer quadratic program with probabilistic constraints. By the "coupling" constraint on $s_{0}$ (Eq. 10), we have to optimize jointly all depot subsystems in the network. This constraint, combining with the quadratic constraints (Eqs. 8-9) and the integer requirement of the decision variables, makes Problem $(P 0)$ very challenging to solve.

To develop an efficient method to solve Problem $(P 0)$, we first apply appropriate linearization techniques to the quadratic terms and the "coupling" constraint to obtain a linear integer stochastic programming (LISP) formulation (§5.1). As noted in the literature, LSIP is also very difficult to solve (Dentcheva, Prékopa and Ruszczynski 2000), and one standard approach is to convert the LISP into a deterministic equivalent formulation by the well-known concept of $p$-efficient points (Prékopa 2003). However, the set of $p$-efficient points can be extremely large, and this concept is associated with a specific probability distribution and thus not applicable here. Yet, by leveraging a unique feature of Problem $(P 0)$, the "finite patient horizon", and Lemma 5, we can derive a "finite" set of deterministic linear constraints equivalent to the fill rate constraints ( $\S 5.2)$. Consequently, we reformulate the original problem into an equivalent linear integer program $(\S 5.3)$. 


\section{$5.1 \quad$ Linearization}

Linearization of the coupling constraint on $s_{0}$ is done by changing the equality sign to an inequality sign, as follows,

$$
s_{0} \geq S-\min \left\{\min _{i}\left\{r_{i}+Q_{i}+\min _{j} s_{i j}\right\}, \min _{j} s_{0 j}\right\} .
$$

The optimal solution shall remain the same regardless of this change because the objective is to minimize the total supply chain cost of the trial. Hence, in the optimal solution, $s_{0}$ will be driven down to $S-\min \left\{\min _{i}\left\{r_{i}+Q_{i}+\min _{j} s_{i j}\right\}, \min _{j} s_{0 j}\right\}$ to achieve the lowest cost possible.

We linearize the quadratic terms $x_{i} Q_{i}$ by the binary representation of $Q_{i}=2^{0} y_{i 0}+2^{1} y_{i 1}+\ldots+$ $2^{n} y_{\text {in }}$, where $n=\lfloor\log (\bar{Q})\rfloor$ and $y_{i 0}, \ldots, y_{i n}$ are binary variables (Billionnet, et. al 2008). Then, $x_{i} Q_{i}=2^{0} x_{i} y_{i 0}+2^{1} x_{i} y_{i 1}+\ldots+2^{n} x_{i} y_{i n}$. Let $Y_{i k}=x_{i} y_{i k}$ where $k=0,1, \ldots, n$, then we have

$$
y_{i}=x_{i} Q_{i}=2{ }^{0} Y_{i 0}+2{ }^{1} Y_{i 1}+\ldots+2{ }^{n} Y_{i n}
$$

Since $Y_{i k}$ is a product of a binary variable $y_{i k}$ and a bounded variable $0 \leq x_{i} \leq S$, it satisfies the following set of inequalities,

$$
\begin{gathered}
Y_{i k} \leq S y_{i k} \\
Y_{i k} \leq x_{i} \\
Y_{i k} \geq x_{i}-S\left(1-y_{i k}\right) .
\end{gathered}
$$

Finally, the constraint $y_{i}=x_{i} Q_{i}$ is equivalent to the following constraints,

$$
\begin{gathered}
y_{i}=2^{0} Y_{i 0}+2^{1} Y_{i 1}+\ldots+2^{n} Y_{i n} \\
Y_{i k} \leq S y_{i k}, \quad k=0,1, \ldots, n \\
Y_{i k} \leq x_{i}, \quad k=0,1, \ldots, n \\
Y_{i k} \geq x_{i}-S\left(1-y_{i k}\right), \quad k=0,1, \ldots, n .
\end{gathered}
$$

\subsection{Chance Constraints}

The fill-rate constraints for the depot $i$ subsystem are

$$
\operatorname{Pr}\left\{s_{i j}-B_{i j}\left(r_{i}, Q_{i}\right)>D_{i j}\left(L_{i j}\right)\right\} \geq \zeta^{\prime}, \quad \forall i j
$$

To convert these chance constraints into a set of deterministic linear constraints, we define $\bar{s}_{i}=$ $\left(r_{i}, Q_{i}, s_{i 1}, s_{i 2}, \ldots, s_{i J_{i}}\right)$ to be an inventory position for the depot $i$ subsystem.

Definition 7 We call $\bar{s}_{i}=\left(r_{i}, Q_{i}, s_{i 1}, s_{i 2}, \ldots, s_{i J_{i}}\right)$ a "minimum inventory position" for the depot $i$ subsystem if it is feasible, i.e., it satisfies all the immediate fill rate constraints for depot $i$ subsystem, and there does not exist a feasible inventory position that is smaller than $\bar{s}_{i}$ component-wisely. 
For each depot subsystem, the number of the minimum inventory positions is bounded from above by $S \times \bar{Q}$ (by Lemma 5). We define the set of minimum inventory positions for depot $i$ subsystem to be $\mathcal{I}_{i}=\left\{\bar{s}_{i}^{(1)}, \bar{s}_{i}^{(2)}, \ldots, \bar{s}_{i}^{\left(n_{i}\right)}\right\}$ where $n_{i}$ is the size of $\mathcal{I}_{i}$. We can rewrite the fill-rate constraints (Eq. 5) for depot $i$ subsystem as follows,

$$
\bar{s}_{i} \geq \mu_{i 1} \bar{s}_{i}^{(1)}+\mu_{i 2} \bar{s}_{i}^{(2)}+\ldots+\mu_{i n_{i}} \bar{s}_{i}^{\left(n_{i}\right)}
$$

where $\mu_{i 1}, \ldots, \mu_{i n_{i}}$ are binary variables, $\mu_{i 1}+\ldots+\mu_{i n_{i}}=1$, and $\geq$ is component-wise. This takes us to the following algorithm for identifying the set of minimum inventory positions for the depot $i$ subsystem.

Step 0. Set $\mathcal{I}_{i}=\emptyset, r_{i}=1, Q_{i}=1$.

Step 1. For each pair of $r_{i}$ and $Q_{i}$ where $r_{i} \leq S$ and $Q_{i} \leq \bar{Q}$, find the smallest $s_{i j}$ at sites that satisfy the immediate fill-rate constraints. Let the output be $\bar{s}_{i}=\left(r_{i}, Q_{i}, s_{i 1}, \ldots, s_{i J_{i}}\right)$. If there does not exist an element in $\mathcal{I}_{i}$ that is smaller than or equal to this output component-wisely, then the output is a minimum inventory position and $\mathcal{I}_{i}=\mathcal{I}_{i} \cup\left\{\bar{s}_{i}\right\}$.

Step 2. Output the set of all minimum inventory positionings $\mathcal{I}_{i}$.

In Step 1, we utilize the two-moment approximation (see, e.g., Graves 1985) to calculate the fill rates, which is proven to be fast and sufficiently accurate. The computational complexity for generating $\mathcal{I}_{i}$ is at most $O\left(S^{2} \times \bar{Q}^{2} \times J_{i}\right)$. In fact, we can generate $\mathcal{I}_{i}$ much faster because given a $Q_{i}$, when $r_{i}$ increases beyond a certain level, the smallest $s_{i j}$ at sites shall remain constant.

\subsection{Integer Programming Reformulation}

We are now ready to present an equivalent linear integer programming reformulation of Problem $(P 0)$. Note that $s_{0 j}$ can be optimized separately (Lemma 5), we let $s_{0 j}^{*}$ be the optimal solution.

$$
\begin{array}{ll}
\min & c\left[s_{0}+\sum_{i}\left(r_{i}+Q_{i}+\sum_{j} s_{i j}\right)+\sum_{j} s_{0 j}^{*}-S\right] \\
& +\sum_{i}\left[K_{i}+v_{i}\left(r_{i}+Q_{i}+\sum_{j} s_{i j}\right)\right]+\sum_{i}\left[K_{i} x_{i}+v_{i} y_{i}\right] \\
\text { s.t. } & \bar{s}_{i} \geq \mu_{i 1} \bar{s}_{i}^{(1)}+\ldots+\mu_{i n_{i}} \bar{s}_{i}^{\left(n_{i}\right)}, \quad \forall i, \\
& \mu_{i 1}+\ldots+\mu_{i n_{i}}=1, \quad \forall i, \quad \forall i, \\
& y_{i}=2^{0} Y_{i 0}+2^{1} Y_{i 1}+\ldots 2^{n} Y_{i n}, \quad, \quad k=1, \ldots, n, \forall i,
\end{array}
$$




$$
\begin{aligned}
& Y_{i k} \leq x_{i}, \quad k=1, \ldots, n, \forall i, \\
& Y_{i k} \geq x_{i}-S\left(1-y_{i k}\right), \quad k=1, \ldots, n, \forall i, \\
& {\left[S-\left(r_{i}+\min _{j} s_{i j}\right)\right] \frac{\sum_{j} \lambda_{i j}}{\sum_{i j} \lambda_{i j}+\sum_{j} \lambda_{0 j}}-Q_{i} \leq y_{i}, \quad \forall i,} \\
& s_{0}+\left\{r_{i}+Q_{i}+s_{i j}\right\} \geq S, \quad \forall i, j, \\
& s_{0}+\min _{j} s_{0 j}^{*} \geq S, \\
& s_{0}, r_{i}, s_{i j}, Q_{i}, x_{i}, y_{i}, Y_{i k} \text { are non-negative integers, } \mu_{i j}, y_{i k} \text { binary, } \quad \forall i, j, k .
\end{aligned}
$$

Problem $(P 1)$ is a linear integer program which can solved by standard integer programming solvers.

\section{Numerical Study}

In this section, we conduct a numerical study on a hypothetical real-life example. The objective is two-fold: (i) to test the efficiency of the solution method and (ii) to develop insights on inventory positioning in clinical trial supply chains. We also demonstrate the potential impact of the site network on the supply chain costs and study the trade-off between inventory overage and recruitment time. The practical intent of the model is to provide decision support to clinical trial supply managers and to facilitate their communication with the study managers regarding the enrollment plans and countries/sites to be include in a trial.

\subsection{Example Details and Computational Performance}

We report the computational performance of the solution method (see §5) on the example in $§ 1.1$. This example is motivated by data ${ }^{4}$ from a real-life Phase III clinical trial that enrolled patients over a 9-month period. Data relevant to this trial's inventory decisions is summarized in Table 2.

By Table 2, the total enrollment rate is 2.18 patients per day. Because the patient horizon, $S$, is 600 patients, the expected recruitment period is 600 patients/2.18 patients per day $\approx 275$ days, which is significantly longer than the importation lead times, and thus the operational characteristics of the system in transient states can be reasonably approximated by their steady-state counterparts. For this numerical study, we assume that all countries use country depots and the intra-country lead time from a depot to a site is one day. Since manufacturing was done outside of all site countries, all distribution goes through their respective country's customs and importation processes. Shipping cost estimates to the country depots are summarized in Table 3.

\footnotetext{
${ }^{4}$ Please note that the data is disguised as requested by the study sponsor.
} 


\begin{tabular}{|l|l|l|l|}
\hline \hline Country & $\begin{array}{l}\text { Importation } \\
\text { Time (days) }\end{array}$ & \# of Sites & $\begin{array}{l}\text { Enrollment Rate by Site (patients per day - } \\
\text { sites separated by commas) }\end{array}$ \\
\hline Latvia & 3 & 4 & $0.02,0.04,0.05,0.08$ \\
Russia & 20 & 4 & $0.03,0.06,0.06,0.28$ \\
Ukraine & 15 & 4 & $0.02,0.04,0.05,0.06$ \\
United States & 10 & 12 & $0.03,0.04,0.05,0.06,0.08,0.08,0.11,0.11$, \\
& 8 & 8 & $0.14,0.14,0.16,0.18$ \\
Poland & 8 & $0.01,0.02,0.04,0.04,0.04,0.06$ \\
\hline
\end{tabular}

Table 2: Enrollment Data for 32 Site Trial conducted in Five Countries.

\begin{tabular}{|l|l|l|l|l|l|}
\hline \hline Country & Latvia & Russia & Ukraine & United States & Poland \\
\hline Fixed Shipping Cost & $\$ 10,000$ & $\$ 40,000$ & $\$ 15,000$ & $\$ 15,000$ & $\$ 10,000$ \\
\hline $\begin{array}{l}\text { Variable Shipping } \\
\text { Cost per package }\end{array}$ & $\$ 200$ & $\$ 500$ & $\$ 750$ & $\$ 500$ & $\$ 400$ \\
\hline
\end{tabular}

Table 3: Shipping Cost Data to Five Countries.

The shipping quantities, $Q_{i}$ 's, are limited by the container size, $\bar{Q}=40$ packages. The cost per drug package, $c$, is estimated to be $\$ 4,000$. The drug cost is high because it is produced via biologic processes as opposed to chemical synthesis.

To solve the example, we choose MOSEK as the integer programming solver. All the computations are done in MATLAB on an Acer laptop running Ubuntu 10.04. The maximum running time for solving any problem instance we analyze is less than 60 seconds.

\subsection{Inventory Positioning}

We now examine the optimal inventory decisions recommended by our model and compare them to the tradition in practice and the existing multi-echelon inventory literature. Figure 3 shows the optimal solution: $s_{0}=583$ and the optimal $\left(r_{i}, Q_{i}\right), s_{i j}$ are the numbers in the triangles representing the depots and sites. The numbers on the arrows from CW to depots are the expected number of shipments (excluding the initial shipment) to the depots given by Eq. (3) (Eq. (3) is quite accurate with an average error of 0.44 and a maximum error of 0.53 for all 112 instances considered in this paper). The optimal solutions of other instances are qualitatively the same.

The optimal solution shows that we may stock a significant amount of inventory at the central 


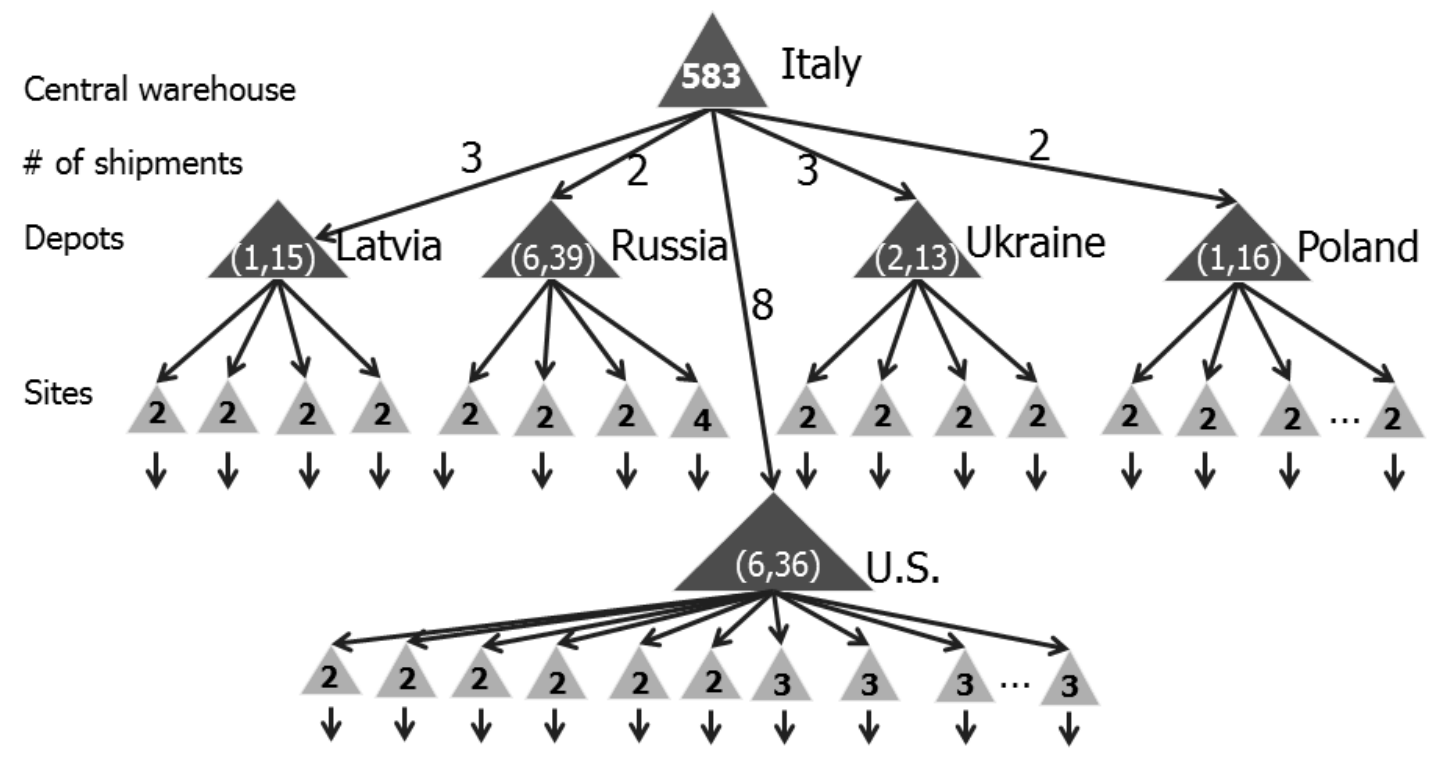

Figure 3: The optimal inventory decisions for $c=\$ 4,000$.

warehouse, and a sizable amount of inventory at country depots relative to the corresponding site level inventory. By doing so, we can achieve significant savings in inventory overage (from the current $100 \%-200 \%$ to 186 packages $\sim 31 \%$ of the patient horizon) while maintaining a high immediate fill rate at all sites and providing a guaranteed supply for the first $S$ (600) patients. The resulting minimum inventory overage and shipping cost is $\$ 1.45$ million.

This solution differs qualitatively from the tradition in practice, see $\S 1.1$ and Peterson, et al. (2004), which aims to save shipping cost by holding most inventory at sites and depots but is suboptimal due to its significant inventory overage. This solution is also qualitatively different from the multi-echelon inventory literature which suggests to hold most of the safety-stock at the lowest echelon. This new insight on inventory positioning comes from the unique features of the clinical trial supply chains (see $\S 1.2)$.

To study the impact of drug cost, we solve the example again but for $c=\$ 2,000, \$ 6,000, \$ 8,000$, $\$ 10,000$. The result is summarized in Figure 4. We observe that as $c$ increases, the reorder points and shipping quantities at country depots tend to decrease which results in a lower inventory overage but a higher shipping cost. The total cost increases significantly as the drug cost increases.

\subsection{Impact of Site Network}

To study how the network of clinical sites affects the inventory overage and total cost, we compare the depth and the breadth strategies where the former has only a few countries but each country 


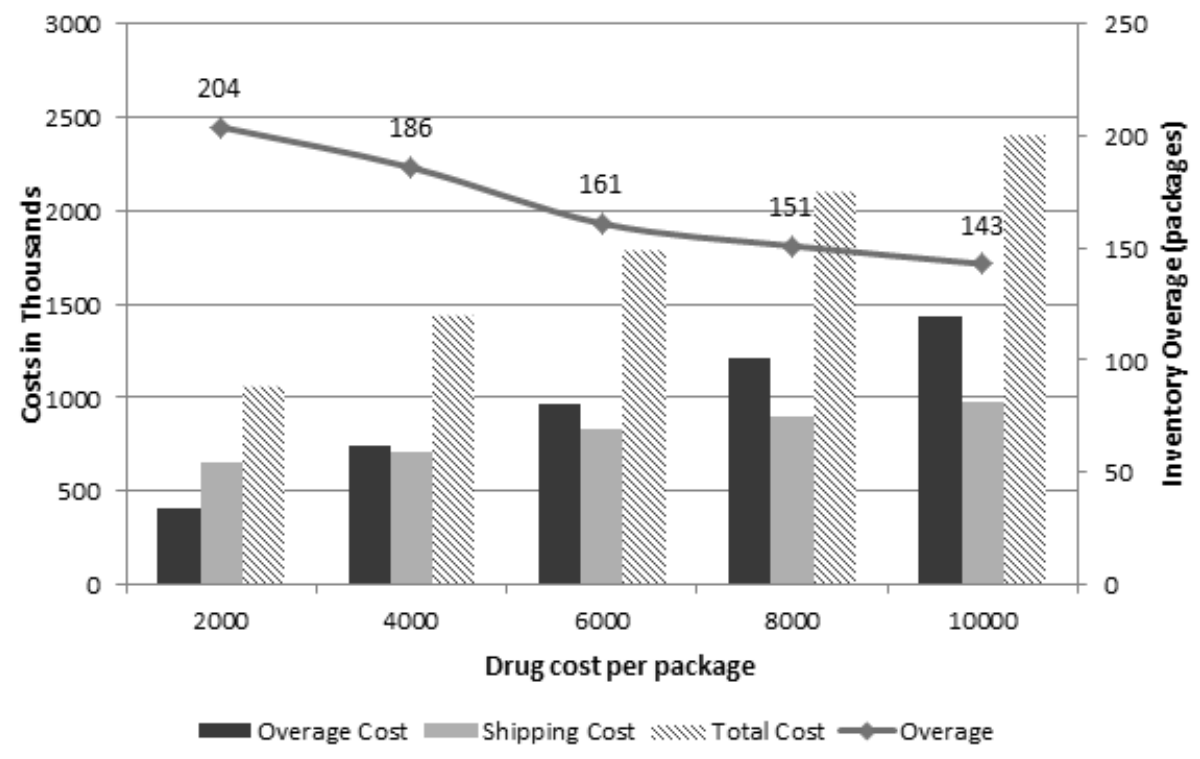

Figure 4: The impact of drug cost.

has many sites, the latter does the opposite - has many countries but each country has fewer sites. Specifically, we consider the following two instances of the example with $c=\$ 4,000$ :

- Depth: the network includes Russia (4 sites), the U.S. (11 sites) and Poland (6 sites).

- Breadth: the network includes Latvia (4 sites), Russia (4 sites), Ukraine (4 sites), the U.S. (6 sites) and Poland (4 sites).

To make these instances comparable, we select sites so that the expected recruitment time is 350 days for both instances.

Figure 5 shows the inventory overage, shipping and total cost of the two instances. As we can see, the depth strategy has a lower inventory overage cost and a lower shipping cost as compared to the breadth strategy. To explain why the depth strategy outperforms the breadth strategy in inventory overage, we pick the U.S. and calculate the inventory per site in each strategy. As we move from the depth strategy to the Breadth strategy, the number of sites in the U.S. decreases from 11 to 6 . Dividing the total inventory $\left(r_{i}+\sum_{j} s_{i j}\right)$ at the depot subsystem of the U.S. by the number of the sites, we find that the inventory per site of the depth (breadth) strategy is 2.91 (3.16, respectively). So the insight is clear: the more sites that a depot serves, the less inventory per site is required, which is the well known risk pooling effect. 


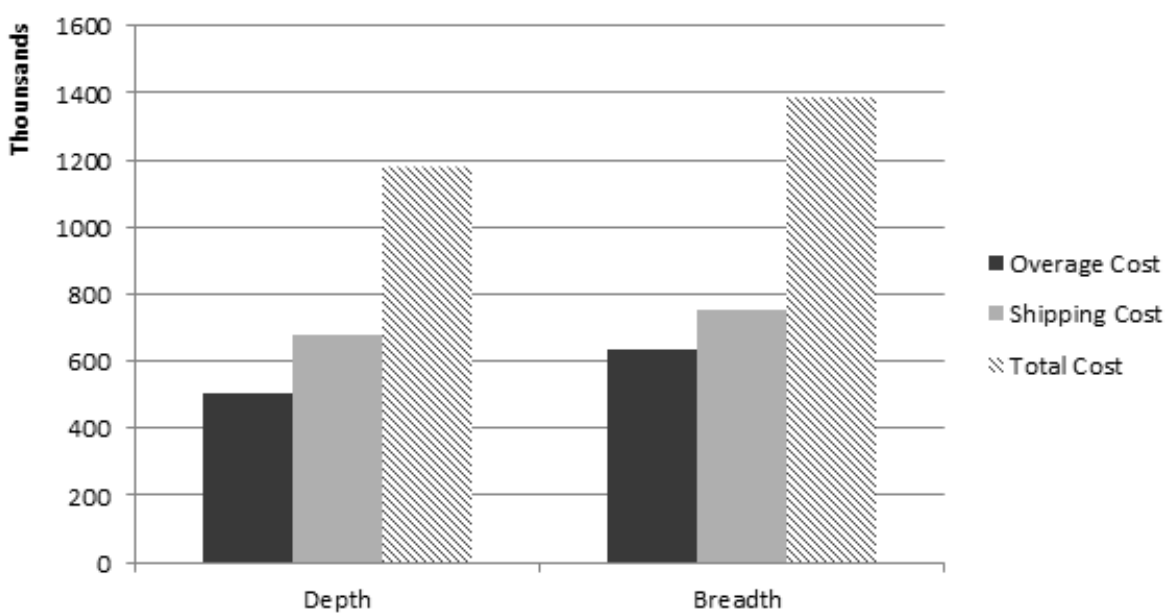

Figure 5: The impact of site network: depth vs. breadth strategies.

\subsection{Overage vs. Recruitment Time}

One important issue that clinical trial management must address is how many countries to include in the trial. Intuitively, the more countries to include in the trial, the shorter the recruitment time but the higher the inventory overage. To study the trade-off between the inventory overage and the expected enrollment time, we take the enrollment data of the motivating example and examine what would happen to the inventory overage, total cost and the expected enrollment time as we add countries into the trial in the order of descending enrollment rates.

The result is summarized in Figure 6 where "2-country" refers to a trial with only the U.S. and Russia, "3-country" refers a trial with the U.S., Russia and Poland, and so on. This figure shows that increasing the number of countries results in sizable decreases in the expected recruitment time but significant increases in the inventory overage. Given roughly the same shipping cost for all cases, the total supply chain cost increases significantly from around $\$ 0.987$ million to about $\$ 1.45$ million while the expected recruitment time decreases from 373 days to 275 days.

For a clinical supply manager, with a limited budget to spend on clinical supplies and a mandate to not delay the trial, being able to show the tradeoff between overages (total supply chain costs) and recruiting time is critical to their discussion with the rest of the clinical trial team. Some countries may be left out of the trial because marginal decreases in recruiting time do not justify the additional costs in supplies. 


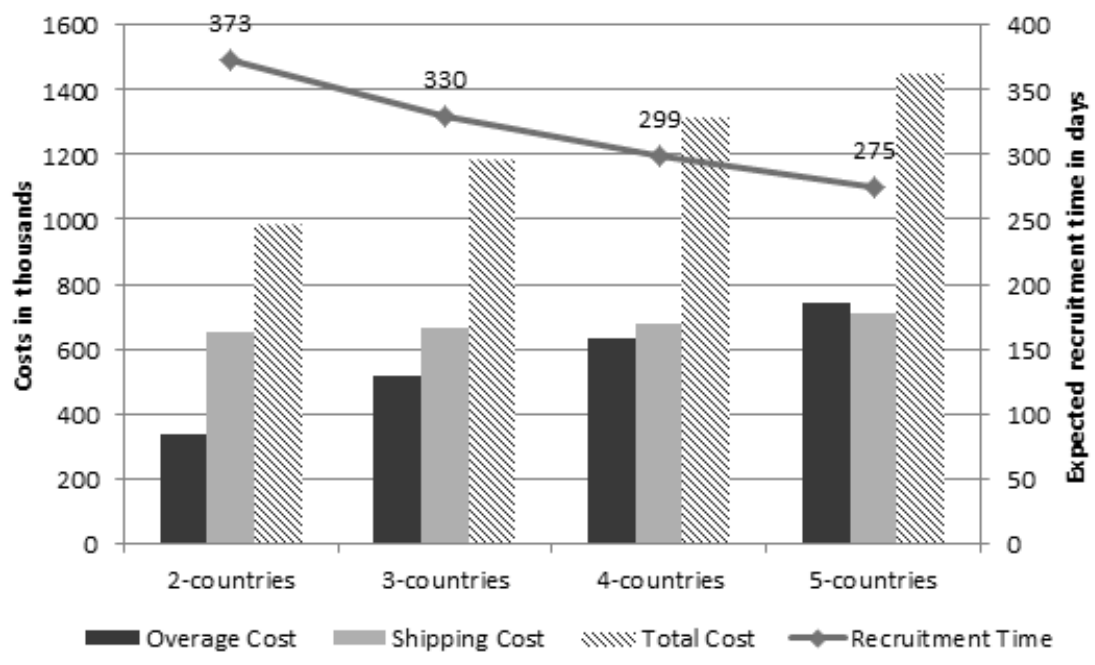

Figure 6: Costs vs. expected recruitment time (in days).

\section{Concluding Remarks}

In this paper, we have illuminated some significant supply issues that today's clinical trials must address. Specifically, getting the right inventory, to the right place, at the right time becomes increasingly difficult in clinical trials as they become more and more globally dispersed. We have constructed a mathematical model for the general clinical trial supply chain based on its unique features to optimally position inventory in support of the trial's service level requirements. We develop a solution method to transform the resulting nonlinear integer program with chance constraints to a deterministic linear integer equivalent. Using a representative example, we develop unique insights on inventory positioning in clinical trial supply chains, and demonstrate the model's potential impact.

Going forward beyond the scope of this paper, clinical trial supply chain research promises to be both fruitful to practitioners because of the high potential impact, and of interest to academicians due to the unique aspects and thus the need for tailored models. The potential in optimizing the drug supply chain has recently been recognized both in academia and in industry. While there is ample work to be done, we suggest the following future research directions:

1. Empirical Studies: There is little systematic empirical study on clinical trial supply management that quantifies the current practice and identifies the factors of impact. One reason is data availability: Data on trial supply costs is often mixed with other types of costs and thus hard to separate. We plan to work with our sponsoring companies to conduct such a study. 
2. Applications of Models: While the model presented in this paper illustrates enormous opportunities to provide value for clinical trial supply chains, applying this model in the real-world practice would help refine and validate it.

3. Integrating Inventory and Project Decisions: It is of great interest to integrate the inventory decisions (discussed in this paper) with clinical trial project decisions, such as country/site selection, design of the study and protocols, and staffing plan to optimize not only the supply chain costs but also the patient and medical expenses.

4. Relaxing the $100 \%$ patient fill rate: while it is ideal (and often the mandate) to guarantee medical supply for the first $S$ patients, it may be overly conservative in some cases. Relaxing this constraint requires a significant modification of the mathematical model in calculating the fill rates and shipping costs as one must consider the possibility of rejecting patients during the trial.

\section{Appendix}

Proof of Lemma 4: Consider the first $S-\left(r_{i}+\min _{j} s_{i j}\right)$ patients recruited. Suppose $N_{i}$ patients are recruited by the depot $i$ subsystem, then the number of orders placed by depot $i$ after the trial starts, $X_{i}$, is given as follows:

$$
\begin{aligned}
X_{i} & =0, \text { if } 0 \leq N_{i}<Q_{i} \\
X_{i} & =1, \text { if } Q_{i}<N_{i}<2 Q_{i} \\
& \cdots \\
X_{i} & =n, \text { if } n Q_{i}<N_{i}<(n+1) Q_{i} .
\end{aligned}
$$

If $N_{i}=n Q_{i}$ for $n \geq 1$, then we have two possibilities: $X_{i}=n$ if the last patient of the first $S-\left(r_{i}+\min _{j} s_{i j}\right)$ patients is recruited by depot $i$ subsystem; $X_{i}=n+1$ otherwise. This is true because depot $i$ orders only when both of the following two conditions are satisfied: (i) its local inventory position drops to $r_{i}$ (ii) there are more than $r_{i}+\min _{j} s_{i j}$ patients left to be recruited. When the $N_{i}$ th patient arrives at depot $i$ subsystem, in the first possibility, condition (i) is satisfied but condition (ii) is not; in the second possibility, both conditions are satisfied, and that is why one more order is placed. The rest of the proof is straightforward.

Proof of Proposition 6: Denote the solution of Problem (17) to be $\mathbf{s}^{\prime}$. Suppose that the propo- 
sition is not true, then there must exist a solution to Problem (16), $\mathbf{s}^{\prime \prime}$, such that

$$
\sum_{j} s_{i j}^{\prime}-\min _{j} s_{i j}^{\prime}>\sum_{j} s_{i j}^{\prime \prime}-\min _{j} s_{i j}^{\prime \prime}
$$

By Lemma 5, we note that given $r_{i}, s_{i j}$ are uniquely determined by the fill rate constraints in both Problem (16) and (17). In addition, $s_{i j}$ is non-increasing in $r_{i}$ (this is clearly true as a higher stocked the regional warehouse only reduces the need of inventory at sites). Thus, Inequality (20) implies $r_{i}^{\prime}<r_{i}^{\prime \prime}$. To see this, we define $\jmath^{\prime}=\operatorname{argmin}_{j}\left\{s_{i j}^{\prime}\right\}, \jmath^{\prime \prime}=\operatorname{argmin}_{j}\left\{s_{i j}^{\prime \prime}\right\}$, and consider two cases:

- Case 1: $\jmath^{\prime}=\jmath^{\prime \prime}$. Then inequality (20) implies $\sum_{j \neq \jmath^{\prime}} s_{i j}^{\prime}>\sum_{j \neq \jmath^{\prime}} s_{i j}^{\prime \prime}$. This is only true when $r_{i}^{\prime}<r_{i}^{\prime \prime}$

- Case 2: $\jmath^{\prime} \neq \jmath^{\prime \prime}$. Rewrite Inequality (20) as

$$
\sum_{j \neq J^{\prime}, J^{\prime \prime}} s_{i j}^{\prime}+s_{i j^{\prime \prime}}^{\prime}>\sum_{j \neq J^{\prime}, J^{\prime \prime}} s_{i j}^{\prime \prime}+s_{i j^{\prime}}^{\prime \prime}
$$

Suppose $r_{i}^{\prime} \geq r_{i}^{\prime \prime}$, then $s_{i j}^{\prime} \leq s_{i j}^{\prime \prime}$ for all $j$, and by the above inequality $s_{i j^{\prime \prime}}^{\prime}>s_{i j^{\prime}}^{\prime \prime}$. Together with $s_{i j^{\prime}}^{\prime \prime} \geq s_{i j^{\prime \prime}}^{\prime \prime}$ (by definition of $j^{\prime \prime}$ ), we must have $s_{i j^{\prime \prime}}^{\prime}>s_{i j^{\prime \prime}}^{\prime \prime}$ which contradicts to the fact that $s_{i j}^{\prime} \leq s_{i j}^{\prime \prime}$ for all $j$. Thus we must have $r_{i}^{\prime}<r_{i}^{\prime \prime}$.

$r_{i}^{\prime}<r_{i}^{\prime \prime}$ implies $s_{i j}^{\prime} \geq s_{i j}^{\prime \prime}$ for all $j$. If there is one $j$ such that $s_{i j}^{\prime}>s_{i j}^{\prime \prime}$, this creates a contradiction to the assumption that $\mathbf{s}^{\prime}$ is optimal for Problem (17). If $s_{i j}^{\prime}=s_{i j}^{\prime \prime}$ for all $j$, this creates a contradiction to Inequality (20). In conclusion, the optimal solution $\mathbf{s}^{\prime}$ for Problem (17) is also optimal for (16).

\section{References}

[1] Abdelkafi, C., H. L. B. Beck, B. David, C. Druck, M. Horoho (2009). Balancing risk and costs to optimize the clinical supply chain - a step beyond simulation. Journal of Pharmaceutical Innovations 4: 96-106.

[2] Andrews, C. (2004). Strategic Value of Clinical Supplies. Pharmaceutical Engineering 24(5).

[3] Axsater, S. (1990). Simple solution procedures for a class of two-echelon inventory problems. Operations Research 38(1) 64-69. 
[4] Axsater, S. (1993a). Continuous review policies for multi-level inventory systems with stochastic demand. In S. Graves, A. Rinnooy Kan, P. Zipkin, (eds.). Logistics of Production and Inventory. Elsevier (North-Holland), Amsterdam. The Netherlands.

[5] Barnett International (2010). Focus on Study Drug. Good Clinical Practice: A Question E Answer Reference Guide 15(15) 2010.

[6] Billionnet, A. and Elloumi, S. and Lambert, A. (2008). Linear Reformulations of Integer Quadratic Programs. Modelling, Computation and Optimization in Information Systems and Management Sciences $\mathbf{1 4} 43-51$.

[7] Byrom, B. (2002). Using IVRS in clinical trial management. Applied Clinical Trials October.

[8] Caggiano, K. E., P. L. Jackson, J. A. Muckstadt, J. A. Rappold (2007). Optimizing Service Parts Inventory in a Multiechelon, Multi-Item Supply Chain with Time-Based Customer Service-Level Agreements. OPERATIONS RESEARCH 55(2) 303-318.

[9] Caglar, D., C. L. Li, D. Simchi-Levi (2004). Two-echelon spare parts inventory system subject to a service constraint. IIE Transactions 36(7) $655-666$.

[10] Dentcheva, D. and Prékopa, A. and Ruszczynski, A. (2000). Concavity and efficient points of discrete distributions in probabilistic programming. Mathematical Programming 89 55-77.

[11] European Commission Enterprise Directorate-General (2000). Manufacture of Investigational medicinal products. Good manufacturing Practices 4(13) July 2003.

[12] Fleischhack, A. (2009). An investigation of clinical trial supply chains. Ph.D Thesis, Department of Supply Chain Management and Marketing Science. Rutgers University - New Newark and New Brunswick. NJ.

[13] Getz, K. A., A. de Bruin (2000). Breaking the development speed barrier: Assessing successful practices of the fastest drug development companies. Drug Information Journal 34 p725 736.

[14] Glickman, S. W., J. G. McHutchison, E. D. Peterson, C. B. Cairns, Robert A. Harrington, Robert M. Califf, Kevin A. Schulman (2009). Ethical and scientific implications of the globalization of clinical research. $N$ Engl J Med 360(8) 816-823.

[15] Graves, S. C. (1985). A multi-echelon inventory model for a repairable item with one-for-one replenishment. Management Science 31(10) 1247-1256. 
[16] Graves, S. C. (1996). A multiechelon inventory model with fixed replenishment intervals. Management Science 42(1) 1-18.

[17] Klim, A. (2010). BRIC by BRIC: Laying a path to success. Pharmaceutical Manufacturing and Packing Sourcer Summer 2010.

[18] Kohner, N. Nemperature Controlled Shipping in Clinical Trials: A Rising Requirement. Almac Group White Paper available at http://www.arradx.com/papers/Papers/Temperature\%20Controlled\%20Shipping \%20in\%20Clinical\%20Trials.pdf and accessed on February 2, 2010.

[19] Le Chevalier, T., D. Brisgand, J.Y. Douillard, J.L. Pujol, V. Alberola, A. Monnier, A. Riviere, P. Lianes, P. Chomy, S. Cigolari (1994). Randomized study of vinorelbine and cisplatin versus vindesine and cisplatin versus vinorelbine alone in advanced non-small-cell lung cancer: results of a European multicenter trial including 612 patients. J Clin Oncol 12(2) 360-367.

[20] Lis, F., P. Gourley, P. Wilson, M. Page (June 1, 2009). Global supply chain management. Applied Clinical Trials Online.

[21] McDonnell, W., H. Mooraj (2009). Case Study: Visualizing Clinical Trial Demand. Industry Value Chain Strategies AMR Research. April 2009.

[22] Muckstadt, J.A. (2005). Analysis and algorithms for service parts supply chains. Springer Verlag.

[23] Oncolytics Biotech Inc. (2003). Oncolytics Biotech Inc. Announces Completion of Manufacturing Process Development for REOLYSIN(R). PR Newswire, February 6, 2003.

[24] Pederson, C., T. Petaja, G. Strauss, H.C. Rumke, A. Poder, J.H. Richardus, B. Spiessens, D. Descamps, K. Hardt, M. Lehtinen, G. Dubin (2007). Immunization of Early Adolescent Females with Human Papillomavirus Type 16 and 18 L1 Virus-Like Particle Vaccine Containing AS04 Adjuvant. Journal of Adolescent Health 40(6) 564-571.

[25] Peterson, M., B. Byrom, N. Dowlman, D. McEntegart (2004). Optimizing clinical trial supply requirements: simulation of computer-controlled supply chain management. Clinical Trials 1(4) 399-412.

[26] Powell, Mark (2010). Presentation by Senior Vice President, Pharmaceutical Development, Bristol-Myers Squibb. Argyle Executive Forum's 2010 Leadership in Pharmaceuticals \& 
Biotechnology conference. June 22, 2010. New York, New York. Transcript available at http://www.argyleforum.com/Thought-Leadership accessed on July 8, 2011.

[27] Prékopa, A. (2003). Probabilistic programming. Handbooks in Operations Research and Management Science 10 267-351.

[28] Ravichandran, N. (1995) Stochastic analysis of a continuous review perishable inventory system with positive lead time and Poisson demand European Journal of Operational Research 84(2) $444-457$.

[29] Roberts, T., T.J. Lynch, B.A. Chabner (2004). The phase III trial in the era of targeted therapy: unraveling the "go or no go" decision. Journal of Clinical Oncology 21(19) 3683-95.

[30] Rowland, C. (2004). Clinical trials seen shifting overseas. International Journal of Health Services 34(3) p555- 556.

[31] Shah, N. (2004). Pharmaceutical supply chains: key issues and strategies for optimisation. Computers and Chemical Engineering 28(6-7) 929-941.

[32] Sherbrooke, C. C. (1968). Metric: A multi-echelon technique for recoverable item control. Operations Research 16(1) 122-141.

[33] Simchi-Levi, D., Y. Zhao. (2005). Safety Stock Positioning in Supply Chains with Stochastic Lead Times. Manufacturing \& Service Operations Management 7(4) 295-318.

[34] Simchi-Levi, D., Y. Zhao (2011). Performance Evaluation of Stochastic Multi-Echelon Inventory Systems: A Survey. Advances in Operations Research 1-34.

[35] Song, J. S. (2000). A note on Assemble-to-Order systems with batch ordering. Management Sciences 46, 739-943.

[36] Svoronos, A., P. Zipkin (1991). Evaluation of one-for-one replenishment policies for multiechelon inventory systems. Management Science 37(1) 68-83.

[37] Thiers, F.A., A.J. Sinskey, E.R. Berndt (2008). Trends in the globalization of clinical trials. Nature Reviews Drug Discovery 7 p13 - 15.

[38] Vallance, P. (2011). Presentation at the Cowen and Company 31st Annual Health Care Conference in Boston. 
Webcast available at http://www.gsk.com/investors/presentations_webcasts.htm/ accessed on March 17, 2011.

[39] Zipkin, P. (1986). Stochastic lead-times in continuous-time inventory models. Naval Research Logistics Quarterly, 33, 763-774.

[40] Zipkin, P. (2000). Foundations of Inventory Management. McGraw Hill, Boston. 